\title{
Photon Momentum Transfer in Single-Photon Double Ionization of Helium
}

\author{
Si-Ge Chen $\odot,{ }^{1}$ Wei-Chao Jiang, ${ }^{2}$ S. Grundmann $\odot,{ }^{3, *}$ F. Trinter, ${ }^{4,5}$ M. S. Schöffler, ${ }^{3}$ T. Jahnke, ${ }^{3, \dagger}$ R. Dörner, ${ }^{3}$ \\ Hao Liang, ${ }^{1}$ Mu-Xue Wang, ${ }^{1}$ Liang-You Peng $\odot{ }^{1,6,7,8, *}$ and Qihuang Gong ${ }^{1,6,7,8}$ \\ ${ }^{1}$ State Key Laboratory for Artificial Microstructure and Mesoscopic Physics, School of Physics, \\ Peking University, Beijing 100871, China \\ ${ }^{2}$ College of Physics and Optoelectronic Engineering, Shenzhen University, Shenzhen 518060, China \\ ${ }^{3}$ Institut für Kernphysik, Goethe-Universität, Max-von-Laue-Strasse 1, 60438 Frankfurt, Germany \\ ${ }^{4}$ FS-PETRA-S, Deutsches Elektronen-Synchrotron (DESY), Notkestrasse 85, 22607 Hamburg, Germany \\ ${ }^{5}$ Molecular Physics, Fritz-Haber-Institut der Max-Planck-Gesellschaft, Faraday 4, 14195 Berlin, Germany \\ ${ }^{6}$ Nano-optoelectronics Frontier Center of the Ministry of Education and Collaborative Innovation Center of Quantum Matter, \\ Beijing 100871, China \\ ${ }^{7}$ Collaborative Innovation Center of Extreme Optics, Shanxi University, Taiyuan 030006, China \\ ${ }^{8}$ Beijing Academy of Quantum Information Sciences, Beijing 100193, China
}

(Received 19 July 2019; revised manuscript received 22 November 2019; published 29 January 2020)

\begin{abstract}
We theoretically and experimentally investigate the photon momentum transfer in single-photon double ionization of helium at various large photon energies. We find that the forward shifts of the momenta along the light propagation of the two photoelectrons are roughly proportional to their fraction of the excess energy. The mean value of the forward momentum is about $\frac{8}{5}$ of the electron energy divided by the speed of light. This holds for fast and slow electrons despite the fact that the energy sharing is highly asymmetric and the slow electron is known to be ejected by secondary processes of shake off and knockout rather than directly taking its energy from the photon. The biggest deviations from this rule are found for the region of equal energy sharing where the quasifree mechanism dominates double ionization.
\end{abstract}

DOI: 10.1103/PhysRevLett.124.043201

The dipole approximation is commonly used in the theoretical treatment of the interaction of photons or light pulses with atoms and molecules. It fails when the wavelength becomes comparable with the atomic size or when the photoelectrons can be accelerated to relativistic velocities at high light intensities. Within the dipole approximation, the photon momentum is neglected and hence the momentum distributions of all produced particles are symmetric upon inversion of the light propagation direction. Triggered by the pioneering experimental work of Smeenk et al. [1] for the single ionization of argon and neon by circularly polarized strong light pulses, the question of the role of the photon momentum has attracted much attention in recent years. At the wavelength of 800 and $1400 \mathrm{~nm}$, Smeenk et al. found that the ion roughly acquires the momenta of those photons needed to overcome the ionization potential $I_{p}$, while the photoelectron takes the momentum roughly equal to the average photoelectron energy divided by the speed of light. More recently, Chelkowski et al. [2] showed theoretically that the photon momentum sharing between the electron and the ion can be more complex. They found different laws for the photon momentum sharing in the perturbative one-photon absorption regime from those in the strong-field multiphoton absorption regime. In the strong-field regime, they reported that the photon momentum sharing law should be slightly modified from the one proposed by Smeenk et al. [1] such that the ion acquires a momentum corresponding to $70 \%$, instead of $100 \%$, of the ionization potential $I_{p}$. This prediction has recently been confirmed experimentally [3]. In the perturbative one-photon absorption regime, they confirmed that the photoelectron can even acquire a momentum larger than the photon momentum as predicted already in the 1930s [4], which leads to the counterintuitive result that the ion is pushed backward by the photon radiation pressure. For the hydrogen atom in different initial states, Seaton [5] theoretically investigated the momentum sharing, pointing out that momentum transfer is important in astrophysics. In more recent works, the problem of photon momentum sharing has been extended from atoms to molecules $[6,7]$ and to the nonsequential double ionization of atoms in the tunneling regime [8].

For the double ionization of atoms, a three-body problem, the presence of the electron-electron correlation makes it hard to investigate the nondipole effects with analytical treatments. However, many novel nondipole effects have recently been identified in experiments on single-photon double ionization (SPDI) of atoms. In particular, for SPDI of helium at extremely large photon energies, the breakdown of one dipole selection rule, i.e., the forbidding of back-to-back emission of electrons with an equal energy, has been found [9-11]. Furthermore, a forward-backward asymmetry of the photoelectron angular distribution along 
the light propagation direction, which was previously known for the single ionization $[12,13]$, has also been predicted in the SPDI [14-16] and the two-photon double ionization (TPDI) $[17,18]$. Recently, the forward-backward asymmetry was experimentally observed in the sequential TPDI [19] of atoms.

Different from the TPDI in the sequential regime, the electron-electron correlation is indispensable in SPDI. How the photon momentum is partitioned among three particles remains an open question. In the present Letter, we address this question in SPDI by theoretically solving the fulldimensional time-dependent Schrödinger equation (TDSE) beyond the dipole approximation and comparing the results with experimental measurements carried out at beam line P04 (PETRA III, DESY, Hamburg). (See [11] for details on the experiment, where a subset of the results for photon energy of $1100 \mathrm{eV}$ is presented.) The data for 385 and $800 \mathrm{eV}$ are shown here for the first time. We find that, similar to the single ionization of hydrogen, the two electrons acquire a total momentum much larger than the transferred photon momentum. In the present high-photon energy regime, the energy sharing between the two electrons is extremely unequal, allowing us to distinguish the primary (fast) electron and the secondary (slow) electron. We identify that the photon momentum allocation between the primary electron and the secondary electron is also extremely unequal and roughly proportional to their fraction of the excess energy.

The present two-electron nondipole TDSE code has been developed based on our previous dipole code for helium, which has been successfully applied in a series of studies on the two-photon double ionization of helium [20-22]. In the time-dependent close-coupling scheme, we discretize the radial coordinates by the finite-element discrete variable representation [23-25], and evolve the wave function using the Lanczos propagation algorithm [26,27]. In the implementation, both the length gauge (LG) and the velocity gauge (VG) have been used to describe the first-order nondipole corrections for the electron-light interaction, and the gauge independence in all the results has been confirmed.

The Hamiltonian in the LG and the VG, respectively, is given by $[7,28,29]$ (atomic units are used throughout this Letter unless otherwise stated),

$$
\begin{aligned}
H_{\mathrm{LG}}= & H_{0}+\sum_{j=1,2} \boldsymbol{r}_{j} \cdot \boldsymbol{E}(t)-\frac{\alpha}{2}\left(\hat{\boldsymbol{k}} \cdot \boldsymbol{r}_{j}\right)\left[\boldsymbol{r}_{j} \cdot \partial_{t} \boldsymbol{E}(t)\right] \\
& -\frac{\alpha}{2} \boldsymbol{L} \cdot[\hat{\boldsymbol{k}} \times \boldsymbol{E}(t)]+O\left(\alpha^{2}\right), \\
H_{\mathrm{VG}}= & H_{0}-\sum_{j=1,2} \mathrm{i} \boldsymbol{A}(t) \cdot \nabla_{j}-\mathrm{i} \alpha\left(\hat{\boldsymbol{k}} \cdot \boldsymbol{r}_{j}\right)\left[\boldsymbol{E}(t) \cdot \nabla_{j}\right] \\
& +\alpha\left(\hat{\boldsymbol{k}} \cdot \boldsymbol{r}_{j}\right)[\boldsymbol{A}(t) \cdot \boldsymbol{E}(t)]+O\left(\alpha^{2}\right),
\end{aligned}
$$

where $H_{0}$ is the field-free Hamiltonian for the two electrons in helium, $\boldsymbol{L}=\boldsymbol{l}_{1}+\boldsymbol{l}_{2}$ is the total angular momentum of the electrons, $\alpha$ is the fine-structure constant, and $\hat{\boldsymbol{k}}$ is the unit vector of the light propagation direction. The LG form of the Hamiltonian is derived through the Power-ZienauWoolley transformation $[30,31]$ from the minimal-coupling Hamiltonian and is kept to the first-order expansion. The VG form of the Hamiltonian is obtained from the minimalcoupling Hamiltonian expanded to the first order with the removal of the $\boldsymbol{A}^{2}(t)$ term [28]. The VG form of the Hamiltonian has been applied in simulations for hydrogen [32] and for helium [33] with high intensities at $10^{17}-10^{20} \mathrm{~W} \mathrm{~cm}^{2}$. In our calculations, the light is assumed to propagate along the $z$ axis. For all the calculations below, the peak intensity is fixed at $10^{10} \mathrm{~W} / \mathrm{cm}^{2}$ and a $\sin ^{2}$ envelope of the pulse has been adopted. Numerical convergences have been guaranteed with maximal numbers of angular momenta $l_{1,2}=9$ and $L=2$ and an average radial grid spacing $\sim 0.14$ a.u. Being freely evolved for a time of at least 10 a.u. after the end of the light pulse, the two-electron wave function is projected onto the uncorrelated symmetrical product of two single-electron scattering states to obtain the joint momentum distribution of the two ejected electrons $P\left(\boldsymbol{k}_{1}, \boldsymbol{k}_{2}\right)$. The momentum spectrum of the ion $P(\boldsymbol{Q})$ can then be derived from $P\left(\boldsymbol{k}_{1}, \boldsymbol{k}_{2}\right)$ by momentum conservation: $P(\boldsymbol{Q})=\int P\left(\boldsymbol{k}_{1}, \boldsymbol{k}_{\text {photon }}-\boldsymbol{k}_{1}-\boldsymbol{Q}\right) d \boldsymbol{k}_{1}$.

In Fig. 1, we present the results of the ion's angularintegrated momentum spectrum (left column) $P\left(Q_{r}\right)$ and the momentum distribution along the light propagation direction $P\left(Q_{z}\right)$ (right column) for light pulses with a lefthanded circular polarization at three different photon energies: 385 (first row), 800 (second row), and $1100 \mathrm{eV}$ (third row). In all the panels, we notice that the theoretical results for the nondipole calculations perfectly agree with those from the experimental measurements, while large discrepancies do exist for the dipole calculations. From the spectra of $P\left(Q_{r}\right)$ presented in the left column of Fig. 1, one observes that the nondipole enhancement is extremely obvious at low momenta, which becomes more and more noticeable with the increase in photon energy. Such a kind of nondipole-induced enhancement at low ion momentum has previously been explained with the quasifree mechanism (QFM) [10,11,34]. The QFM, which contributes to the pure-quadrupole yield, can be taken as a benchmark to examine the calculations. In the spectra of $P\left(Q_{z}\right)$ shown in the right column of Fig. 1, one finds that the peak of both the nondipole calculation and the experimental measurement shifts towards the opposite direction of the light propagation, while the distribution of the dipole calculation is symmetric about the line $Q_{z}=0$. In fact, such a nondipole shift in $P\left(Q_{z}\right)$ reflects that the photon momentum transferred to the ion is negative, which is similar to the hydrogen single-photon ionization.

Single-photon double ionization is frequently pictured as being composed of two steps: the interaction of the light field with one of the electrons and a subsequent step in which the second electron is set free, mediated by the 


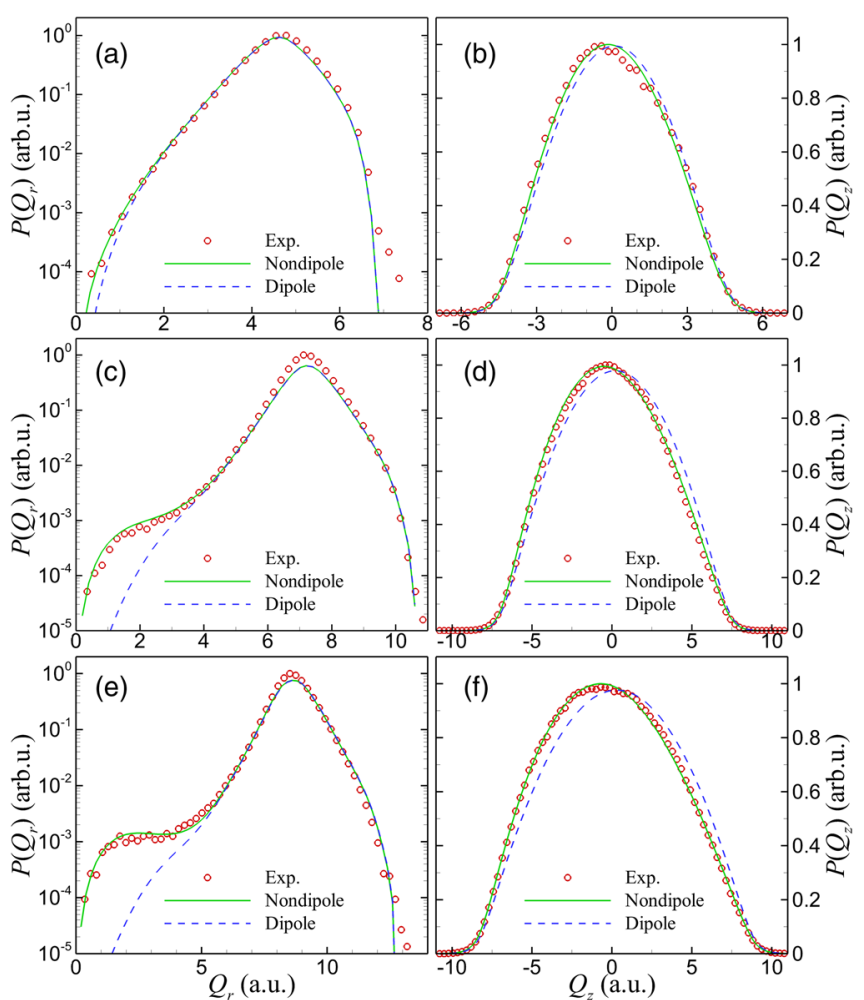

FIG. 1. The yield of $\mathrm{He}^{2+}$ as a function of the magnitude of its momentum $Q_{r}$ for photon energies of (a) 385, (c) 800, and (e) $1100 \mathrm{eV}$. The distribution of the $\mathrm{He}^{2+}$ momentum $Q_{z}$ along the light propagation direction for photon energy at (b) 385 , (d) 800, and (f) $1100 \mathrm{eV}$, in which $Q_{x}$ and $Q_{y}$ have been integrated over. The experimental data have been normalized to the theoretical calculations (normalized) for a best fitting.

electron-electron correlation as manifested in the shake-off [35-37], the knock-out [37], or the quasifree mechanism $[10,11,34]$. The forward-backward asymmetry of the ion along the light propagation is useful to check the validity of the dipole-quadrupole interference [12]. On the other hand, the situation is more intricate for electrons, as the energy of one individual electron can take any value from $E_{1}=0$ to $E_{1}=E_{e x}$. Therefore, the question arises of how the average momentum in $z$ direction $\left\langle k_{z, 1}\right\rangle\left(E_{1}\right)$ depends on the energy of the electron $E_{1}$ for a given photon energy, where $\left\langle k_{z, 1}\right\rangle\left(E_{1}\right)$ is defined by

$$
\left\langle k_{z, 1}\right\rangle\left(E_{1}\right)=\frac{1}{P\left(E_{1}\right)} \iint d \hat{k}_{1} d \boldsymbol{k}_{2} k_{1} \cos \theta_{1} P\left(\boldsymbol{k}_{1}, \boldsymbol{k}_{2}\right),
$$

in which $P\left(E_{1}\right)=\iint d \hat{k_{1}} d \boldsymbol{k}_{2} P\left(\boldsymbol{k}_{1}, \boldsymbol{k}_{2}\right)$ the probability of single electron with energy $E_{1}$ in SPDI. In Figs. 2(a)-2(c), $\left\langle k_{z, 1}\right\rangle\left(E_{1}\right)$ and $P\left(E_{1}\right)$, together with their product $\left\langle k_{z, 1}\right\rangle\left(E_{1}\right) P\left(E_{1}\right)$, are shown for 385,800 , and $1100 \mathrm{eV}$, respectively. We find that $\left\langle k_{z, 1}\right\rangle\left(E_{1}\right)$ roughly follows the straight line $\left\langle k_{z, 1}\right\rangle\left(E_{1}\right)=\frac{8}{5} E_{1} / c$, which one would expect if the electron of energy $E_{1}$ had been ejected by single
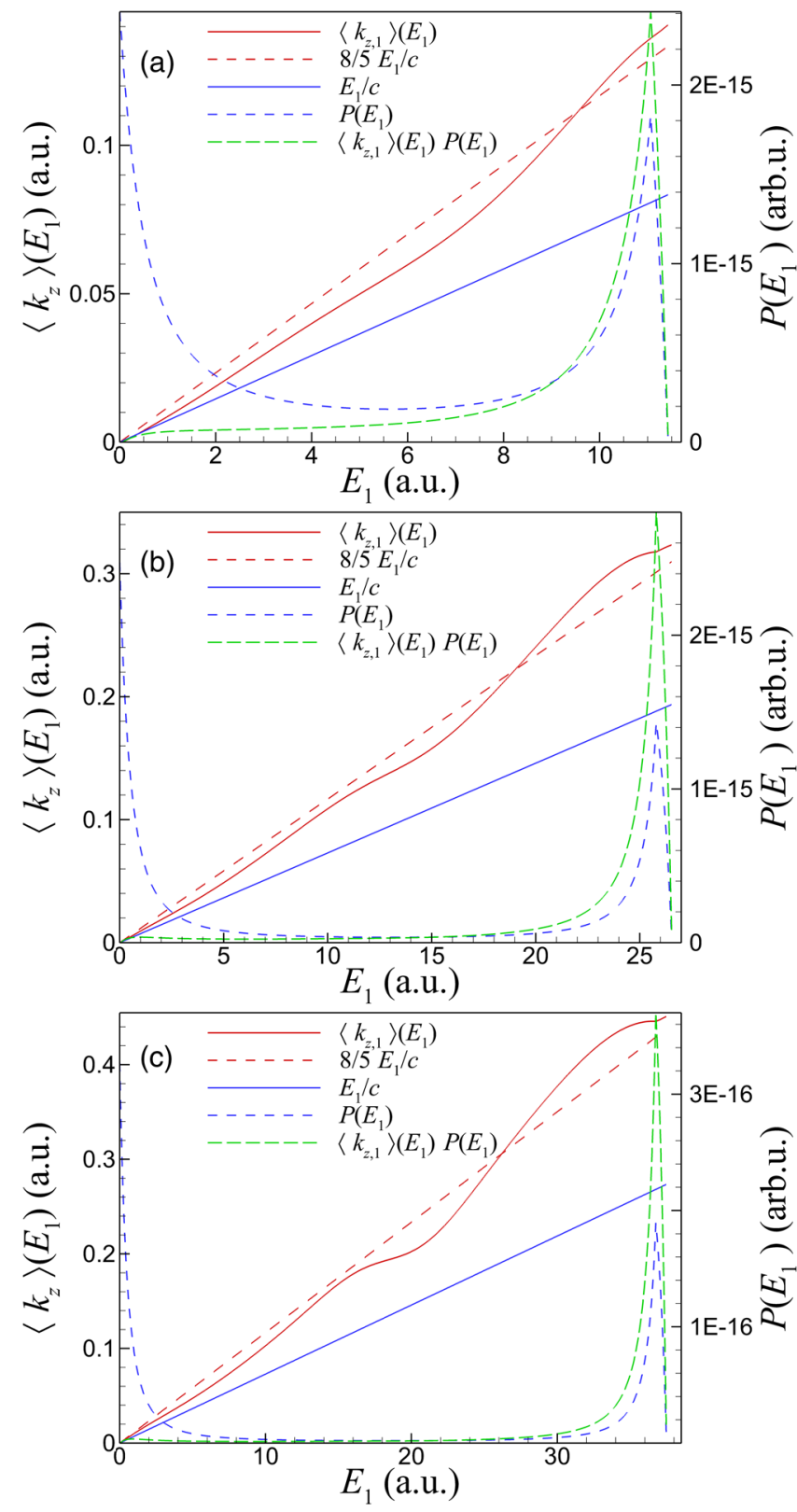

FIG. 2. The average momentum of electron $\left\langle k_{z, 1}\right\rangle\left(E_{1}\right)$, probability of single electron with specific energy $P\left(E_{1}\right)$, and their product as a function of the energy of a single electron $E_{1}$ for photon energy at 385 (a), 800 (b), and $1100 \mathrm{eV}$ (c). The red dashed line shows a momentum of $\frac{8}{5} E_{1} / c$ and the blue line shows $E_{1} / c$.

ionization at the respective smaller photon energy. Thus in SPDI the $\frac{8}{5}$ of the photon momentum is shared among the two electrons roughly according to their energy. The largest deviation from this simple scaling law is observed at equal energy sharing, where $\left\langle k_{z, 1}\right\rangle\left(E_{1}\right)$ deviates from $\frac{8}{5} E_{1} / c$, being more twisted towards $E_{1} / c$ with increasing photon energy. This goes along with the increasing contribution of the QFM with increasing photon energy (see the left column in Fig. 1). $E_{1} / c$ is the scaling one would expect if the photon momentum couples to the electron and not to the nucleus. 
This supports the intuitive explanation that the QFM is a process where the electron pair absorbs the photon while the nucleus remains a spectator [9-11] absorbing none of the photon's linear momentum, energy, or angular momentum. The QFM contributes only very close to equal energy sharing. For extremely unequal energy sharing the slow electron is known to be dominantly set free via shake off while slow electrons with energies of more than $10 \mathrm{eV}$ are more likely to be produced by knockout. Despite this change from shake off to knockout as function of electron energy sharing we find an almost constant slope of $\left\langle k_{z, 1}\right\rangle\left(E_{1}\right)$ for the energy sharing $E_{1} /\left(E_{1}+E_{2}\right)<\frac{1}{3}$. This suggests that other than for the QFM, for knockout and shake off the electrons distribute the $\frac{8}{5}$ of the photon momentum according to their energy.

To test these theoretical predictions from Fig. 2 we compare them in an aggregated fashion to the experimental findings. The average momentum acquired by one electron can be calculated through

$$
\frac{1}{2}\left\langle k_{z}^{\text {total }}\right\rangle=\iint d \boldsymbol{k}_{1} d \boldsymbol{k}_{2} k_{1} \cos \theta_{1} \tilde{P}\left(\boldsymbol{k}_{1}, \boldsymbol{k}_{2}\right),
$$

where $\theta$ is the angle between the electron momentum and the photon momentum, and $\tilde{P}\left(\boldsymbol{k}_{1}, \boldsymbol{k}_{2}\right)=P\left(\boldsymbol{k}_{1}, \boldsymbol{k}_{2}\right) / P_{\text {total }}$ is the normalized differential probability of the double ionization, with $P_{\text {total }}=\iint d \boldsymbol{k}_{1} d \boldsymbol{k}_{2} P\left(\boldsymbol{k}_{1}, \boldsymbol{k}_{2}\right)$ being the probability of SPDI. The average momentum of the ion $\left\langle Q_{z}\right\rangle$ is simply calculated by the momentum conservation law, $\left\langle Q_{z}\right\rangle=\omega / c-\left\langle k_{z}^{\text {total }}\right\rangle$. Please note that the average momenta $\left\langle k_{z}^{\text {total }}\right\rangle$ and $\left\langle Q_{z}\right\rangle$ and the momentum distribution along the light propagation direction are insensitive to the ellipticity of the light, which can be analytically proven in the frame of the perturbation theory for the single-photon double ionization of $\mathrm{He}$ [15] and is also numerically confirmed in our present study for SPDI. Furthermore, we can divide the electrons in the slow and fast half and define

$$
\begin{aligned}
\left\langle k_{\text {fast }}\right\rangle & =2 \iint d \boldsymbol{k}_{1} d \boldsymbol{k}_{2} k_{1} \cos \theta_{1} \eta\left(k_{1}-k_{2}\right) \tilde{P}\left(\boldsymbol{k}_{1}, \boldsymbol{k}_{2}\right), \\
\left\langle k_{\text {slow }}\right\rangle & =2 \iint d \boldsymbol{k}_{1} d \boldsymbol{k}_{2} k_{1} \cos \theta_{1} \eta\left(k_{2}-k_{1}\right) \tilde{P}\left(\boldsymbol{k}_{1}, \boldsymbol{k}_{2}\right),
\end{aligned}
$$

in which $\eta(x)$ is the Heaviside step function, and the factor 2 comes from the identity of the two electrons.

In Fig. $3, \frac{1}{2}\left\langle k_{z}^{\text {total }}\right\rangle,\left\langle Q_{z}\right\rangle,\left\langle k_{\text {fast }}\right\rangle$, and $\left\langle k_{\text {slow }}\right\rangle$ are plotted as a function of $E_{\mathrm{ex}} / c=\left(\omega-I_{p}\right) / c$, where $I_{p}=2.9037$ a.u. is the double ionization potential for helium, for photon energies from 99 to $1100 \mathrm{eV}$. It clearly shows (both theoretically and experimentally) that when the photon energy is high enough, the radiation pressure pushes ions in the opposite direction of the photon momentum, which is interesting in the astrophysics of stellar atmospheres [5].

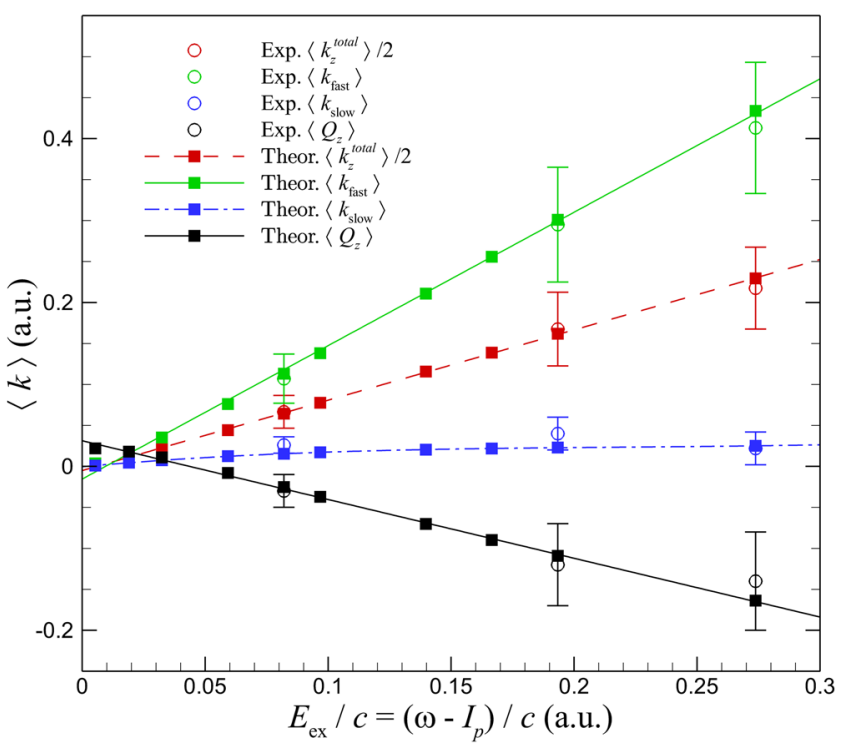

FIG. 3. The average momenta for the electrons and the ion along the direction of the light propagation as functions of the excess energy divided by the light speed, i.e., $E_{\mathrm{ex}} / c=$ $\left(\omega-I_{p}\right) / c$. See the text for the definitions of these observables.

$\left\langle k_{z}^{\text {total }}\right\rangle$ and $\left\langle Q_{z}\right\rangle$ linearly depend on $E_{\text {ex }} / c$, and a linear fitting of the data points respectively gives

$$
\begin{aligned}
\left\langle k_{z}^{\text {total }}\right\rangle & \approx 1.72 \frac{E_{\mathrm{ex}}}{c}-0.01 \\
\left\langle Q_{z}\right\rangle & \approx-0.72 \frac{E_{\mathrm{ex}}}{c}+\frac{I_{p}}{c}+0.01 .
\end{aligned}
$$

It is worth pointing out that Eqs. (7) and (8) are quite similar to the known relation in the one-photon single ionization of hydrogen [2],

$$
\begin{aligned}
\left\langle k_{z}^{\text {single }}\right\rangle & =\frac{8}{5} \frac{E_{\mathrm{ex}}}{c}, \\
\left\langle Q_{z}^{\text {single }}\right\rangle & =-\frac{3}{5} \frac{E_{\mathrm{ex}}}{c}+\frac{I_{p}}{c},
\end{aligned}
$$

except for two differences. First, according to Eq. (7), when $E_{\text {ex }}=0,\left\langle k_{z}^{\text {total }}\right\rangle \neq 0$, which conflicts with the expectation that the photoelectron with zero kinetic energy must lead to zero value of $\left\langle k_{z}^{\text {total }}\right\rangle$. We emphasize that this unphysical result originates from the nonlinear dependence of $\left\langle k_{z}^{\text {total }}\right\rangle$ on $E_{\text {ex }} / c$. Our linear fitting formula is only effective for high excess energies $E_{\mathrm{ex}}$. Secondly, the total linear momentum transferred to the two electrons in SPDI is slightly higher than that transferred to the single electron in the onephoton single ionization of hydrogen. The slope of $\left\langle k_{z}^{\text {total }}\right\rangle$ to $E_{\text {ex }} / c$ in the present SPDI is approximately 1.72 , while the slope in the single-photon single ionization of hydrogen has been analytically proven to be 1.60 . 
The linear fitting of $\left\langle k_{\text {fast }}\right\rangle$ gives

$$
\left\langle k_{\text {fast }}\right\rangle \approx 1.63 \frac{E_{\text {ex }}}{c}-0.015 \text {. }
$$

The slope 1.63 of $\left\langle k_{\text {fast }}\right\rangle$ is close to the slope of $\left\langle k_{z}^{\text {single }}\right\rangle$ for the hydrogen atom. For comparison, the slope we extracted from the single ionization is 1.58 in the two-electron He. The shake-off mechanism is known to contribute more than the knock-out for photon energy larger than $300 \mathrm{eV}$ (cf., Fig. 1 of Ref. [36]). As we roughly estimate, the slope of $\left\langle k_{\text {fast }}\right\rangle$ for shake off is also close to 1.6 (for more details, see Supplemental Material [38]).

In summary, we have theoretically and experimentally studied the photon momentum partition among the two electrons and the ion in SPDI of helium. Our work is the first experimental confirmation of the scaling laws for the photon momentum sharing in the perturbation regime. Excellent agreement has been achieved in the theoretical calculations and the experimental measurements in both the differential distribution of the ion momentum and the linear momentum sharing at large photon energies. In particular, we have found that besides the region of equal energy sharing where the QFM mechanism dominates, the forward shift of the slow and fast electron is given roughly by $\frac{8}{5}$ of its respective energy divided by the speed of light. This is the forward momentum that the electron would also have gained if emitted in single ionization at the relatively lower photon energy. This proportionality of the forward shift to the electron energy leads to the fact that the fast electron which carries most of the photon's energy also has the biggest forward momentum shift while the shake-off and knock-out electrons show only little forward shift. Our present study opens a door to study the complicated photon momentum transfer in those processes where multiple particles are produced by absorbing a few photons.

This work is partially supported by the National Key R\&D Program of China (Grant No. 2018YFA0306302) and by the National Natural Science Foundation of China (NSFC) under Grants No. 11725416, No. 11961131008, and No. 11804233. The experimental work was supported by DFG and BMBF. We acknowledge DESY (Hamburg, Germany), a member of the Helmholtz Association HGF, for the provision of experimental facilities. Parts of this research were carried out at PETRA III and we would like to thank the staff for excellent support during the beam time.

*grundmann@atom.uni-frankfurt.de

jahnke@atom.uni-frankfurt.de

*liangyou.peng@pku.edu.cn

[1] C. T. L. Smeenk, L. Arissian, B. Zhou, A. Mysyrowicz, D. M. Villeneuve, A. Staudte, and P. B. Corkum, Phys. Rev. Lett. 106, 193002 (2011).
[2] S. Chelkowski, A. D. Bandrauk, and P. B. Corkum, Phys. Rev. Lett. 113, 263005 (2014).

[3] A. Hartung, S. Eckart, S. Brennecke, J. Rist, D. Trabert, K. Fehre, M. Richter, H. Sann, S. Zeller, K. Henrichs, G. Kastirke, J. Hoehl, A. Kalinin, M. S. Schöffler, T. Jahnke, L. Ph. H. Schmidt, M. Lein, M. Kunitski, and R. Dörner, Nat. Phys. 15, 1222 (2019).

[4] A. Sommerfeld and G. Schur, Ann. Phys. (Leipzig) 396, 409 (1930).

[5] M. J. Seaton, J. Phys. B 28, 3185 (1995).

[6] S. Chelkowski and A. D. Bandrauk, Phys. Rev. A 97, 053401 (2018).

[7] H. Liang, M.-X. Wang, X.-R. Xiao, Q. Gong, and L.-Y. Peng, Phys. Rev. A 98, 063413 (2018).

[8] A. Emmanouilidou, T. Meltzer, and P. Corkum, J. Phys. B 50, 225602 (2017).

[9] A. G. Galstyan, O. Chuluunbaatar, Y. V. Popov, and B. Piraux, Phys. Rev. A 85, 023418 (2012).

[10] M. S. Schöffler, C. Stuck, M. Waitz, F. Trinter, T. Jahnke, U. Lenz, M. Jones, A. Belkacem, A. L. Landers, M. S. Pindzola, C. L. Cocke, J. Colgan, A. Kheifets, I. Bray, H. SchmidtBöcking, R. Dörner, and T. Weber, Phys. Rev. Lett. 111, 013003 (2013).

[11] S. Grundmann, F. Trinter, A. W. Bray, S. Eckart, J. Rist, G. Kastirke, D. Metz, S. Klumpp, J. Viefhaus, L. Ph. H. Schmidt, J. B. Williams, R. Dörner, T. Jahnke, M. S. Schöffler, and A. S. Kheifets, Phys. Rev. Lett. 121, 173003 (2018).

[12] J. W. Cooper, Phys. Rev. A 47, 1841 (1993).

[13] B. Krässig, E. P. Kanter, S. H. Southworth, R. Guillemin, O. Hemmers, D. W. Lindle, R. Wehlitz, and N. L. S. Martin, Phys. Rev. Lett. 88, 203002 (2002).

[14] A. Y. Istomin, N. L. Manakov, A. V. Meremianin, and A. F. Starace, Phys. Rev. Lett. 92, 063002 (2004).

[15] A. Y. Istomin, N. L. Manakov, A. V. Meremianin, and A. F. Starace, Phys. Rev. A 71, 052702 (2005).

[16] A. Y. Istomin, A. F. Starace, N. L. Manakov, A. V. Meremianin, A. S. Kheifets, and I. Bray, J. Phys. B 39, L35 (2006).

[17] A. N. Grum-Grzhimailo and E. V. Gryzlova, J. Phys. B 45, 215602 (2012).

[18] E. V. Gryzlova, A. N. Grum-Grzhimailo, S. I. Strakhova, and M. Meyer, J. Phys. B 46, 164014 (2013).

[19] M. Ilchen et al., Nat. Commun. 9, 4659 (2018).

[20] Z. Zhang, L.-Y. Peng, M.-H. Xu, A. F. Starace, T. Morishita, and Q. Gong, Phys. Rev. A 84, 043409 (2011).

[21] W. C. Jiang, L. Y. Peng, W. H. Xiong, and Q. Gong, Phys. Rev. A 88, 023410 (2013).

[22] W.-C. Jiang, J.-Y. Shan, Q. Gong, and L.-Y. Peng, Phys. Rev. Lett. 115, 153002 (2015).

[23] T. N. Rescigno and C. W. McCurdy, Phys. Rev. A 62, 032706 (2000).

[24] B. I. Schneider and L. A. Collins, J. Non-Cryst. Solids 351, 1551 (2005).

[25] M. J. Rayson, Phys. Rev. E 76, 026704 (2007).

[26] T. J. Park and J. C. Light, J. Chem. Phys. 85, 5870 (1986).

[27] C. Leforestier and A. Guldberg, J. Comput. Phys. 94, 59 (1991).

[28] M. X. Wang, X. R. Xiao, H. Liang, S. G. Chen, and L. Y. Peng, Phys. Rev. A 96, 043414 (2017). 
[29] R. Anzaki, Y. Shinohara, T. Sato, and K. L. Ishikawa, Phys. Rev. A 98, 063410 (2018).

[30] J. Fiutak, Can. J. Phys. 41, 12 (1963).

[31] D. L. Andrews, G. A. Jones, A. Salam, and R. G. Woolley, J. Chem. Phys. 148, 040901 (2018).

[32] M.-X. Wang, H. Liang, X.-R. Xiao, S.-G. Chen, W.-C. Jiang, and L.-Y. Peng, Phys. Rev. A 98, 023412 (2018).

[33] H. Bachau and M. Dieng, Eur. Phys. J. D 73, 123 (2019).

[34] M. Y. Amusia, E. G. Drukarev, V. G. Gorshkov, and M. O. Kazachkov, J. Phys. B 8, 1248 (1975).

[35] J. C. Levin, I. A. Sellin, B. M. Johnson, D. W. Lindle, R. D. Miller, N. Berrah, Y. Azuma, H. G. Berry, and D.-H. Lee, Phys. Rev. A 47, R16 (1993).

[36] T. Schneider, P. L. Chocian, and J. M. Rost, Phys. Rev. Lett. 89, 073002 (2002).

[37] A. Knapp, A. Kheifets, I. Bray, T. Weber, A. L. Landers, S. Schössler, T. Jahnke, J. Nickles, S. Kammer, O. Jagutzki, L. Ph. H. Schmidt, T. Osipov, J. Rösch, M. H. Prior,
H. Schmidt-Böcking, C. L. Cocke, and R. Dörner, Phys. Rev. Lett. 89, 033004 (2002).

[38] See Supplemental Material at http://link.aps.org/supplemental/ 10.1103/PhysRevLett.124.043201 for more details, which includes Refs. [39-42].

[39] A. L. Godunov, C. T. Whelan, H. R. J. Walters, V. S. Schipakov, M. Schöffler, V. Mergel, R. Dörner, O. Jagutzki, L. Ph. H. Schmidt, J. Titze, and H. Schmidt-Böcking, Phys. Rev. A 71, 052712 (2005).

[40] M. S. Schöffler, O. Chuluunbaatar, Y. V. Popov, S. Houamer, J. Titze, T. Jahnke, L. Ph. H. Schmidt, O. Jagutzki, A. G. Galstyan, and A. A. Gusev, Phys. Rev. A 87, 032715 (2013).

[41] V. Mergel, R. Dörner, K. Khayyat, M. Achler, T. Weber, O. Jagutzki, H. J. Lüdde, C. L. Cocke, and H. Schmidt-Böcking, Phys. Rev. Lett. 86, 2257 (2001).

[42] E. G. Drukarev and A. I. Mikhailov, High-Energy Atomic Physics (Springer, Cham, Switzerland, 2016). 